\title{
Effects of the components of two antimicrobial emulsions on food-borne pathogens
}

\author{
J.P. Ferreira ${ }^{a}$, D. Alves ${ }^{a}$, O. Neves ${ }^{a}$, J. Silva ${ }^{\text {a }}$, P.A. Gibbs ${ }^{\text {a,b }}$, P.C. Teixeira ${ }^{\mathrm{a}, *}$ \\ ${ }^{a}$ CBQF/Escola Superior de Biotecnologia - U.C.P., Rua Dr. António Bernardino de Almeida, 4200-072 Porto, Portugal \\ ${ }^{\mathrm{b}}$ Leatherhead Food International, Randalls Road, Leatherhead, Surrey KT22 7RY, UK
}

Keywords:

Microemulsion

Nanoemulsion

Antimicrobial

Bactericidal

Detergent

Food pathogens

\section{A B S T R A C T}

Several recent reports in the literature show that some microemulsions and nanoemulsions have interesting antimicrobial properties. However, those studies lacked appropriate controls showing the action of the isolated components at similar concentrations. In this study we demonstrate that the bactericidal effects of two previously studied emulsions are due predominantly to one of their components. In fact, the bioactivity of those compounds, namely tributyl phosphate and $n$-pentanol, is diminished when incorporated in the dispersed oil phases. Therefore, we suggest that future work regarding the antimicrobial action of emulsions include controls similar to the ones carried out in this research.

\section{Introduction}

In recent years, a few research groups have presented data regarding the antimicrobial properties of certain microemulsions and nanoemulsions. Nanoemulsions are fine emulsions with dispersed phase aggregates within the submicron region. They are only kinetically stable and are mostly opaque (Tadros, Izquierdo, Esquena, \& Solans, 2004). Microemulsions can present several different structures, but they are kinetically and thermodynamically stable, and are transparent or translucent (Lawrence \& Rees, 2000).

One nanoemulsion for which a considerable amount of data has been presented is known as BCTP. This is an oil-in-water O/W type emulsion, with soybean oil, Triton X-100 and tributyl phosphate as non-aqueous components. Hamouda and Baker (2000) observed that it has bactericidal properties against Gram-positive but not against Gram-negative species. It has also been shown to be sporicidal in different dilutions (Hamouda et al., 1991, 2001), antiviral against enveloped viruses (Donovan et al., 2001; Hamouda et al., 2001), and fungistatic (Hamouda et al., 2001). This research group, whose main focus is in human health applications, has also tested a large number of alternative emulsions, as can be found in a recent patent application (Baker, Hamouda, Shih \& Andrzej, 2003).

Another research team has worked with a microemulsion of ethyl oleate, using Tween 80 as emulsifier and $n$-pentanol as coemulsifier (herein designated TEOP). They demonstrated a strong bactericidal effect in suspension cultures of both Staphylococcus

\footnotetext{
* Corresponding author. Tel./fax: +351 225580095.

E-mail address: pcteixeira@esb.ucp.pt (P.C. Teixeira).
}

aureus and Pseudomonas aeruginosa (Al-Adham, Khalil, Al-Hmoud, Kierans, \& Collier, 2000). Later, the activity of the microemulsion was evaluated against biofilms of $P$. aeruginosa (Al-Adham, AlHmoud, Khalil, Kierans, \& Collier, 2003).

Based on these quite interesting results, we extended the study of the microbicidal properties of both BCTP and TEOP against a large range of common food-borne pathogens, in both the suspension form and in biofilms (Teixeira et al., 2007).

Curiously enough, in none of the above reports we find studies that evaluate the contributions of the different components of the emulsions for the observed activity. In the case of BCTP, it is reported that Triton X-100 and tributyl phosphate are needed for their microbicidal action (Baker, 2005; Hamouda et al., 1999), but without quantitative evidence. Therefore, we carried out a careful analysis of the bactericidal action of the different components of the above two emulsions individually, in order to evaluate the true effects of the emulsification, and/or synergistic effects among different components. As target microorganisms, we selected Listeria monocytogenes, because of its sensitivity to BCTP, and $S$. aureus and Escherichia coli, because of their considerable resistance to this emulsion (Teixeira et al., 2007).

\section{Materials and methods}

Bacterial strains and growth conditions

S. aureus NCTC 1803, L. monocytogenes and E. coli 0157:H7 (VT negative) were generously supplied by the culture collection of Escola Superior de Biotecnologia, Porto, Portugal. Bacterial cultures 
were maintained at $-80^{\circ} \mathrm{C}$ in tryptone soy broth (TSB) (LabM, Bury, UK) with $30 \% \mathrm{v} \mathrm{v}^{-1}$ glycerol. Working cultures were grown on tryptone soy agar (TSA) (LabM, Bury, UK). TSB was inoculated with colonies from TSA plates and incubated at $37^{\circ} \mathrm{C}$ for $24 \mathrm{~h}$. This culture was then used to inoculate fresh TSB, and incubated for another $24 \mathrm{~h}$ at $37^{\circ} \mathrm{C}$.

\section{Preparation of emulsions}

The BCTP nanoemulsion was prepared according to Hamouda et al. (1999) and Baker, Hamouda, Shih, and Andrzej (2003). The oil phase components are soybean oil $\left(16 \% \mathrm{v} \mathrm{v}^{-1}\right.$ of the total emulsion), tri- $n$-butyl phosphate (2\%), and Triton X-100 (2\%). These components were mixed and kept for $1 \mathrm{~h}$ at $86^{\circ} \mathrm{C}$. After this, the water phase was added, and the mixture was emulsified with a high sheer laboratory mixer (Ultra-Turrax T25, Janke \& Kunkel GMBH, Staufen, Germany) for 5 min, or with continuous sonication (LabSonic U, B.Braun, Melsungen, Germany) for a similar time interval. BCTP containing $0.25 \%\left(\mathrm{w} \mathrm{v}^{-1}\right)$ of cetylpyridinium chloride (BCTP-CPC) was prepared in the same way as BCTP, with the addition of CPC to the aqueous phase before emulsification.

The TEOP microemulsion was prepared according to Al-Adham et al. (2000). This microemulsion is also an O/W type, with the oil phase comprised of ethyl oleate ( $3 \% \mathrm{v} \mathrm{v}^{-1}$ of the total emulsion), $n$ pentanol (6\%), and Tween 80 (15\%). After adding the water to the oil phase, emulsification was promoted simply by vortex mixing.

Aqueous solutions of each component were prepared at the same concentration as in the emulsion (or at other indicated dilution). For oils, their mixtures with water were vortex-mixed or subjected to high shear mixing, as when preparing the above emulsions, and immediately used.

\section{Kinetics of killing}

Overnight bacterial cultures were added at $1 \% \mathrm{v} \mathrm{v}^{-1}$ to the tested emulsions or solutions. In most experiments, at 5, 15 and $30 \mathrm{~min}, 1 \mathrm{ml}$ samples were taken from those inoculated suspensions and immediately diluted in $9 \mathrm{ml}$ of sterile Ringer's solution at room temperature. For viable counts, $100 \mu \mathrm{l}$ samples were spread onto TSA and the plates were incubated at $37^{\circ} \mathrm{C}$ for $24 \mathrm{~h}$. Control experiments were carried out in the same way, substituting deionized water for the emulsion or solutions.

At least three independent experiments were carried out for each set of conditions. Error bars in figures represent the standard deviation of those experiments. When not visible, error bars are within the size of the data points.

\section{Results and discussion}

\section{Antimicrobial action of BCTP and its isolated components}

Fig. 1 shows the action of the different components of BCTP, at the same concentration as in the emulsion, against $S$. aureus. A maximum of 1-log reduction in the counts is observed with the tributyl phosphate solution after $30 \mathrm{~min}$. Previously, we have shown with a similar experiment that BCTP emulsion leads to less than 1-log reduction in S. aureus (Teixeira et al., 2007). Therefore, the resistance of this bacterium against BCTP and its isolated components is now corroborated.

The data obtained with the same solutions and oil dispersion but with L. monocytogenes is presented in Fig. 2. This organism shows considerable sensitivity towards tributyl phosphate, leading to a 6-log reduction in $15 \mathrm{~min}$. This is in agreement with our data with BCTP emulsion, and its dilutions up to $1 / 100$, against this microorganism (Teixeira et al., 2007). It is interesting to observe

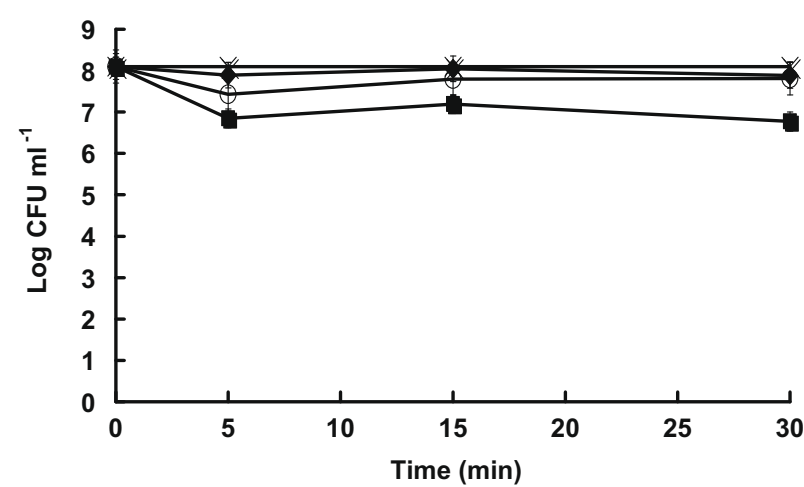

Fig. 1. Changes in $\log \mathrm{CFU} \mathrm{ml} \mathrm{m}^{-1}$ observed with the different components of BCTP emulsion acting on S. aureus: oil ( ); tributyl phosphate ( $\mathbf{\square})$; Triton X-100 (O); control $(x)$

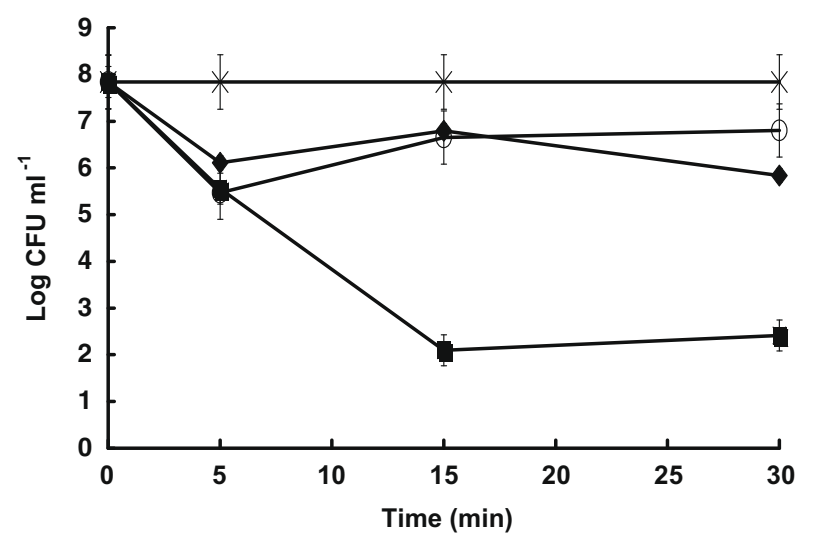

Fig. 2. Changes in $\log \mathrm{CFU} \mathrm{ml} \mathrm{m}^{-1}$ observed with the different components of BCTP emulsion acting on L. monocytogenes: oil ( $)$; tributyl phosphate ( $\mathbf{\square})$; Triton X-100 (O); control $(\times)$.

in Fig. 2 that a fine dispersion of soybean oil-in-water lead to $c a$. $2-\log$ reduction in the counts.

The difference in results between $S$. aureus and $L$. monocytogenes indicates that not all Gram-positive bacteria are susceptible to BCTP (or its components), as previously stated (Hamouda \& Baker, 2000; Hamouda et al., 2001). Furthermore, we conclude that the antimicrobial activity of this emulsion is due mainly to tributyl phosphate. In fact, compounds with similar structures, such as certain alkyl phosphonates, are known to have bacteriostatic properties (Vaution, 1983). In order to corroborate this conclusion, we compared the bactericidal activity against Listeria of BCTP emulsion with an analogous emulsion devoid of tributyl phosphate (Fig. 3). This last emulsion led to $c a$. 1-log reduction in the viable cells in $30 \mathrm{~min}$, while BCTP led to a 3-log reduction. Comparing this result for BCTP with the one for the solution of tributyl phosphate (Fig. 2), we conclude additionally that the antimicrobial action of this compound is reduced when incorporated in the emulsion.

\section{Antimicrobial action of BCTP-CPC and CPC solution}

In order to enlarge the spectrum of activity of BCTP and other emulsions, the incorporation of a cationic surfactant in the formulations has been tested (Baker, 2005). We have used cetylpyridinium chloride (CPC), a well known antimicrobial agent. Figs. 4a-4c show the action of BCTP containing $0.25 \%\left(\mathrm{w} \mathrm{v}^{-1}\right)$ CPC (BCTPCPC) and also the action of an aqueous solution of CPC at the same concentration, against S. aureus, L. monocytogenes and E. coli. Addition of CPC did not improve significantly the bactericidal effect of 


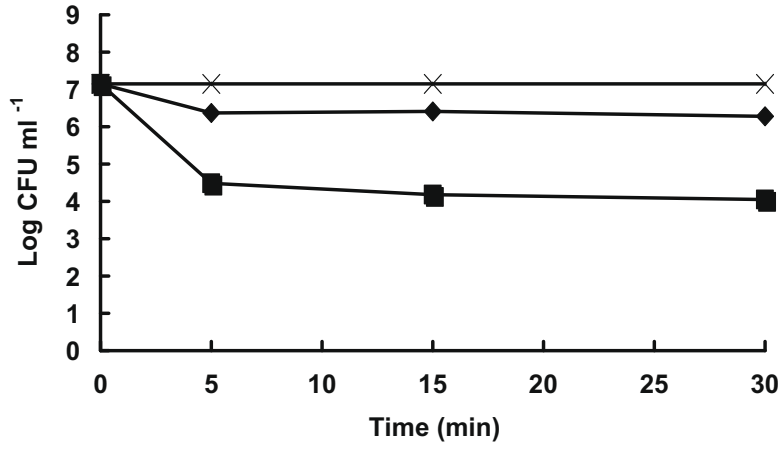

Fig. 3. Changes in log CFU ml ${ }^{-1}$ observed with BCTP emulsion ( $\left.\boldsymbol{\square}\right)$, similar emulsion devoid of tributyl phosphate $(\checkmark)$, and control $(\times)$, acting on L. monocytogenes.

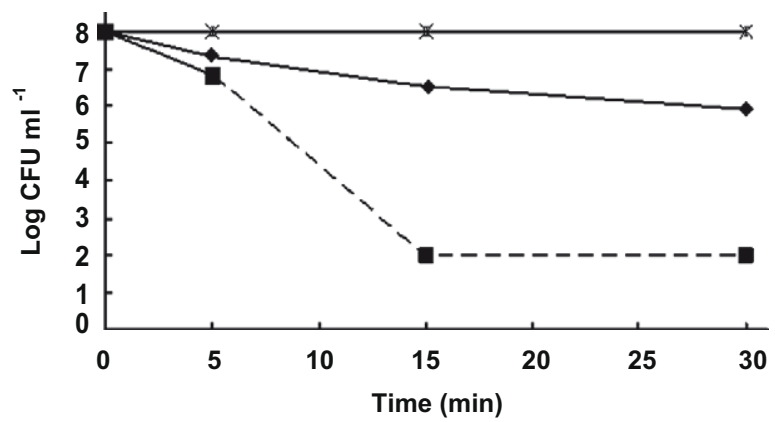

Fig. 4a. Changes in log CFU ml ${ }^{-1}$ observed with BCTP-CPC emulsion ( $\left.\downarrow\right)$, solution of $\mathrm{CPC}(\boldsymbol{\square})$, and control $(\times)$, acting on S. aureus. When no colonies where recovered, the limit of detection ( $100 \mathrm{CFU} \mathrm{ml}^{-1}$, using the 1:10 dilution) is represented, with different time points linked by broken lines.

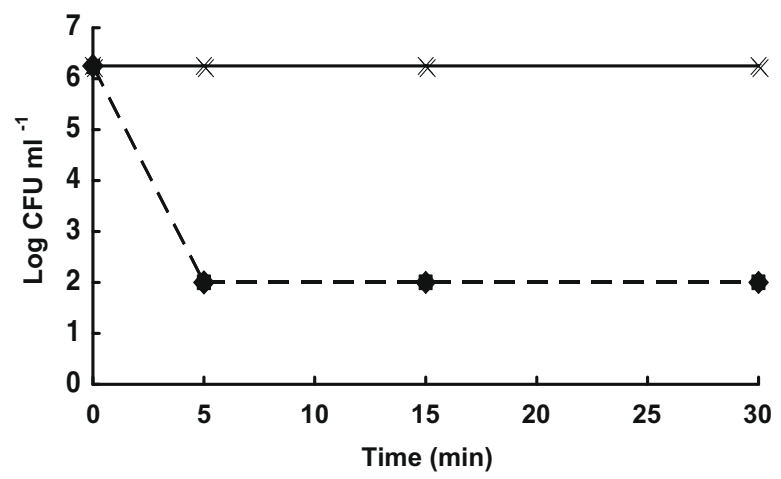

Fig. 4b. Changes in log CFU ml ${ }^{-1}$ observed with BCTP-CPC emulsion $(\bullet)$, solution of CPC $(\mathbf{\square})$, and control $(\times)$, acting on L. monocytogenes. When no colonies where recovered, the limit of detection (CFU ml${ }^{-1}$, using the 1:10 dilution) is represented, with different time points linked by broken lines.

BCTP emulsion on S. aureus or E. coli, a conclusion based on this data and previous one (Teixeira et al., 2007). However, the bactericidal action on Listeria was considerably improved (vide Figs. 3 and $4 \mathrm{~b})$. The solution of CPC alone had a strong biocidal activity against $E$. coli and $S$. aureus, showing that the incorporation of the compound in the emulsion reduces its activity.

\subsection{Antimicrobial action of TEOP and its components}

Previous work has shown that this microemulsion kills almost instantaneously a large group of both Gram-positive and Gramnegative bacteria (Al-Adham et al., 2000; Teixeira et al., 2007).

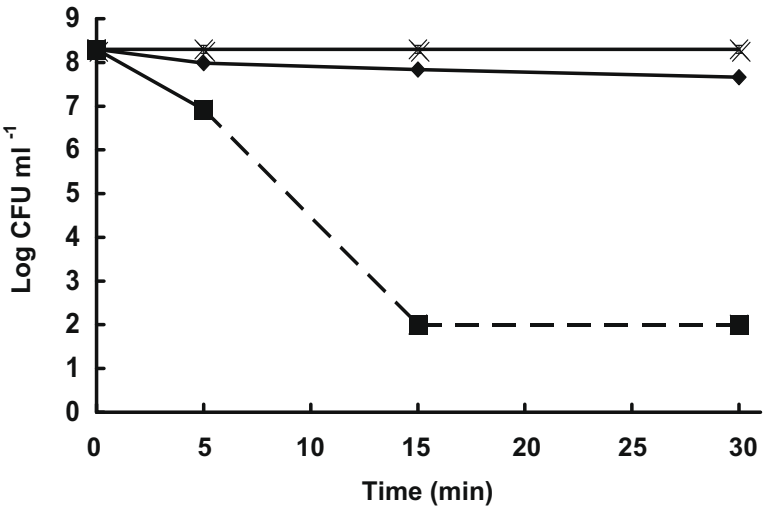

Fig. 4c. Changes in log CFU ml ${ }^{-1}$ observed with BCTP-CPC emulsion $(\bullet)$, solution of CPC ( $\mathbf{\square})$, and control $(\times)$, acting on E. coli. When no colonies where recovered, the limit of detection ( $100 \mathrm{CFU} \mathrm{ml}^{-1}$, using the 1:10 dilution) is represented, with different time points linked by broken lines.

However, it has been observed that a 10 -fold dilution of the emulsion leads to loss of bactericidal effect (Teixeira et al., 2007). Figs. 5 and 6 show the action of each component of that emulsion against $S$. aureus and L. monocytogenes, respectively. S. aureus was affected by only one of the components, $n$-pentanol. Listeria was also inactivated by this alcohol and, albeit more slowly, also by a dispersion of ethyl oleate in water. Therefore, we conclude that the bactericidal activity of TEOP is due mainly to $n$-pentanol, with no differences in the kinetics of killing of a $6 \%$ solution of this alcohol and the whole emulsion.

Furthermore, we evaluated the action of a 10 -fold dilution of the $n$-pentanol solution $\left(0.6 \% \mathrm{v} \mathrm{v}^{-1}\right.$ final concentration), and we observed that both $S$. aureus and Listeria are resistant to this concentration of the alcohol (data not shown), in agreement with our previous findings with the diluted TEOP emulsion (Teixeira et al., 2007).

The bactericidal properties of alcohols have been known for a long time, although most studies have been centred on short-chain (particularly ethanol) and long-chain members (Harold, 1970; Ingram \& Buttke, 1984; Kubo, Muroi, \& Kubo, 1995). It is known that the alcohols disrupt cell membranes, leading to leakage of ions and metabolites. The antimicrobial power of different alcohols is, therefore, related to their capacity of interaction with the cell membranes (Ingram \& Buttke, 1984). It is possible that mediumchain alcohols, such as $n$-pentanol, interact more strongly with the membranes than shorter and longer-chain ones.

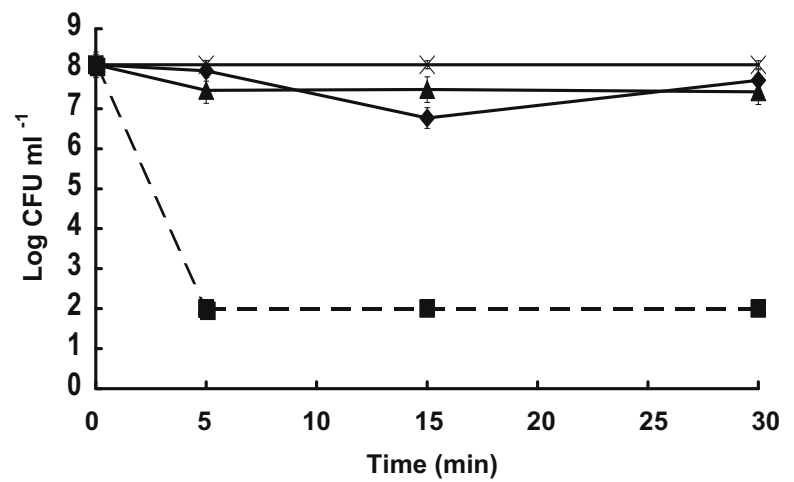

Fig. 5. Changes in $\log \mathrm{CFU} \mathrm{m} \mathrm{m}^{-1}$ observed with the different components of TEOP emulsion, action on S. aureus: ethyl oleate $(\checkmark)$; Tween 80 ( $\mathbf{\Delta})$; pentanol $(\boldsymbol{\square})$; control $(\times)$. When no colonies where recovered, the limit of detection $\left(100 \mathrm{CFU} \mathrm{ml}{ }^{-1}\right.$, using the $1: 10$ dilution) is represented, with different time points linked by broken lines. 


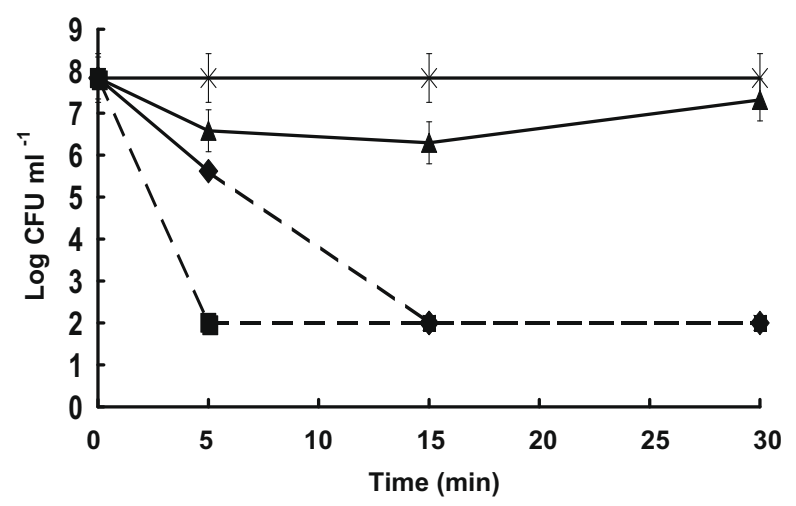

Fig. 6. Changes in $\log \mathrm{CFU} \mathrm{ml} \mathrm{m}^{-1}$ observed with the different components of TEOP emulsion, action on L. monocytogenes: ethyl oleate ( $)$; Tween 80 ( $\mathbf{\Delta}$ ); pentanol ( $\mathbf{\square})$; control $(x)$. When no colonies where recovered, the limit of detection ( $100 \mathrm{CFU} \mathrm{ml}^{-1}$, using the $1: 10$ dilution) is represented, with different time points linked by broken lines.

\section{Discussion}

Several recent publications have noted the potential antimicrobial activity of nanoemulsions and microemulsions. In all of those studies, there are no data that clearly evaluate the contribution of the individual components. Supposedly, the emulsions will have particular affinity to, and activity on, the pathogens' membranes (Al-Adham et al., 2000; Hamouda \& Baker, 2000). For example, BCTP is said to be lysogenic (Baker, 2005). Here we show that the bactericidal activity of two of the previously tested emulsions is due predominantly to one of their components. In fact, the activity of those agents is reduced when incorporated in the emulsions. This means that the availability of such agents for attacking the cell membrane is diminished. Both tributyl phosphate, which has surfactant properties, and $n$-pentanol, a co-surfactant used in microemulsions, are likely to be present at the interface of the emulsion oil droplets, which lowers their affinity for the lipids in the cell membranes.

It can be suggested that the potential toxic effects of antimicrobial agents can be diminished when incorporated in emulsions.
This statement needs to be evaluated with studies comparing the toxicity of the emulsions with that of solutions of those compounds with equivalent biocidal activity. Therefore, we strongly suggest that future work concerning the antimicrobial action of emulsions be accompanied by studies regarding the effects of their isolated components.

\section{References}

Al-Adham, I. S. I., Khalil, E., Al-Hmoud, N. D., Kierans, M., \& Collier, P. J. (2000). Microemulsions are membrane-active, antimicrobial, self-preserving systems. Journal of Applied Microbiology, 89, 32-39.

Al-Adham, I. S. I., Al-Hmoud, N. D., Khalil, E., Kierans, M., \& Collier, P. J. (2003). Microemulsions are highly effective anti-biofilm agents. Applied Microbiology, 36, 97-100.

Baker, J. R. Jr., Hamouda, T., Shih, A., \& Andrzej, M. (2003). Non-toxic antimicrobial compositions and methods of use. US patent $6,559,189$.

Baker, J. R. Jr. (2005). Antimicrobial nanoemulsion composition and methods. WO 2005/030172 A1.

Donovan, B. W., Reuter, J. D., Cao, Z., Myc, A., Johnson, K. J., \& Baker, J. R. Jr., (2001). Prevention of murine influenza A virus pneumonitis by surfactant nanoemulsions. Antiviral Chemistry and Chemotherapy, 11, 41-49.

Hamouda, T., \& Baker, J. R. Jr., (2000). Antimicrobial mechanism of action of surfactant lipid preparations in enteric Gram-negative bacilli. Journal of Applied Microbiology, 89, 397-403.

Hamouda, T., Hayes, M. M., Cao, Z., Tonda, R., Johnson, K., Wright, D. C., et al. (1999). A novel surfactant nanoemulsion with broad-spectrum sporicidal activity against Bacillus species. Journal of Infection Diseases, 180, 1939-1949.

Hamouda, T., Myc, A., Donovan, B., Shih, A. Y., Reuter, J. D., \& Baker, J. R. Jr., (2001). A novel surfactant nanoemulsion with a unique non-irritant topical antimicrobial activity against bacteria, enveloped viruses and fungi. Microbiology Research $156,1-7$.

Harold, F. M. (1970). Antimicrobial agents and membrane function. Advances in Microbial Physiology, 4, 45-104.

Kubo, I., Muroi, H., \& Kubo, A. (1995). Structural functions of antimicrobial longchain alcohols and phenols. Bioorganic Medicinal Chemistry, 3, 873-880.

Lawrence, M. J., \& Rees, G. D. (2000). Microemulsion-based media as novel drug delivery systems. Advanced Drug Delivery Reviews, 45, 89-121.

Ingram, L. O‘N., \& Buttke, T. M. (1984). Effects of alcohols on microorganisms. Advances in Microbial Physiology, 25, 253-300.

Tadros, T., Izquierdo, P., Esquena, J., \& Solans, C. (2004). Formation and stability of nano-emulsions. Advances in Colloid Interface Science, 108-109, 303-318.

Teixeira, P. C., Leite, G. M., Domingues, R. J., Silva, J., Gibbs, P. A., \& Ferreira, J. P. (2007). Antimicrobial effects of a microemulsion and a nanoemulsion on enteric and other pathogens and biofilms. International Journal of Food Microbiology, $118,15-19$.

Vaution, C. (1983). Les agents de surface. Classification. In F. Puisieux \& M. Seiller (Eds.), Les Systèmes Dispersés I Agents de Surface et Émulsions. Technique et Documentation (pp. 5-50). Paris: Lavoisier. 